\title{
Cardiopulmonary arrest induced by atlantoaxial dislocation with subarachnoid hemorrhage: a case report and review of the literature
}

\author{
Hiroshi Kageyama ${ }^{1} \cdot$ Kousuke Kakumoto $^{1} \cdot$ Hiroki Yasuoka $^{2} \cdot$ Hirohiko Arimoto $^{1} \cdot$ Yukoh Ohara $^{3}$
}

Received: 26 October 2019 / Revised: 27 November 2019 / Accepted: 2 December 2019

(C) International Spinal Cord Society 2019

\begin{abstract}
Introduction Spinal cord injury (SCI) with atlantoaxial dislocation (AAD) is often fatal. We present the case of a resuscitated patient with AAD and traumatic subarachnoid hemorrhage (SAH) at the craniovertebral junction (CVJ).

Case presentation We present the case of an 84-year-old man who suffered an observed cardiopulmonary arrest. Cardiopulmonary resuscitation was initiated and spontaneous circulation returned. In the emergency room, the patient's Glasgow Coma Scale was 3 (E1V1M1). No spontaneous respiration was noted. Neuroimaging revealed SAH at the CVJ. Contrastenhanced computed tomography (CT) revealed a vessel running through the left $\mathrm{C} 2 / 3$ intervertebral foramen into the spinal canal. The ventral space of spinal cord revealed contrast enhancement. Angiography revealed extravasation from the spinal branch of the left vertebral artery, without venous filling. It did not appear to be a vascular malformation with an arteriovenous shunt, but rather a traumatic laceration of the artery. Plain CT and CT angiography suggested AAD. Magnetic resonance imaging revealed injury to the medulla oblongata and upper cervical spinal cord, with AAD and retrodental subligamentous hemorrhage. We embolized the branch of the left vertebral artery and performed a $\mathrm{C} 1$ laminectomy. The patient moved his extremities postoperatively.

Discussion This was a case of injury to the medulla oblongata and upper cervical spinal cord due to AAD with SAH. This is the first report of resuscitated case of traumatic AAD with SAH in the CVJ. Traumatic AAD should be included in the differential diagnosis in case of SAH in CVJ, which may be misdiagnosed as intrinsic SAH.
\end{abstract}

\section{Introduction}

Spinal cord injury (SCI) with atlantoaxial dislocation (AAD) is often fatal, and resuscitation is rare [1]. We treated a resuscitated patient with traumatic $\mathrm{AAD}$ and traumatic subarachnoid hemorrhage $(\mathrm{SAH})$ at the craniovertebral junction $(\mathrm{CVJ})$, following low-energy trauma. At the emergency room, we confused the condition with intrinsic SAH. There have been no similar case reports in the past. We describe this case and discuss the relevant literature.

Hiroshi Kageyama

kageyamahiroshi29@gmail.com

1 Department of Neurosurgery, Shin-Kuki General Hospital, Kuki, Saitama, Japan

2 Department of Orthopedic surgery, Tokorozawa Meisei Hospital, Tokorozawa, Saitama, Japan

3 Department of Neurosurgery, Juntendo University, Tokyo, Japan

\section{Case presentation}

In this report, we present the case of an 84-year-old man with a history of diabetes mellitus who suffered from cerebral infarction 3 months prior to admission. While he and his son slept in the same room, his son noticed a loud knocking sound, followed by an apparent cardiopulmonary arrest in the patient's bed. His son called an ambulance and performed cardiopulmonary resuscitation. Spontaneous circulation returned and the patient was transported to the emergency room.

On arrival, the patient's Glasgow Coma Scale was 3 (E1V1M1). No spontaneous respiration was noted. There were no signs of trauma on the whole-body surface. He was intubated and artificially ventilated. Laboratory findings did not show any abnormalities in blood count or biochemical results, apart from slight anemia.

Initial plain chest X-ray and noncontrast head and body computed tomography (CT) scans were performed. No pathogenetic lesions were detected in the thorax or abdomen that could explain the cardiopulmonary arrest. 
Fig. 1 CT and MRI (FLAIR) show SAH at the CVJ. a CT, b MRI (FLAIR).

Fig. 2 CT angiographies did not reveal any aneurysm. a They revealed a vessel running through the left $\mathrm{C} 2 / 3$ intervertebral foramen into the spinal canal (white arrows). b The ventral space of spinal cord presented a contrastenhanced effect ventrally (white arrows).

Fig. 3 Angiography of the left vertebral artery revealed extravasation from the spinal branch of the left vertebral artery, without venous filling in all phases. It did not appear to be a vascular malformation with an arteriovenous shunt, but rather was a traumatic laceration of the artery. a AP view of the angiography in arterial phase. b AP view in late arterial phase. c AP view in venous phase. d Lateral view of the angiography. e AP view of three-dimensional digital subtraction angiography. f Lateral view of the threedimensional digital subtraction angiography.
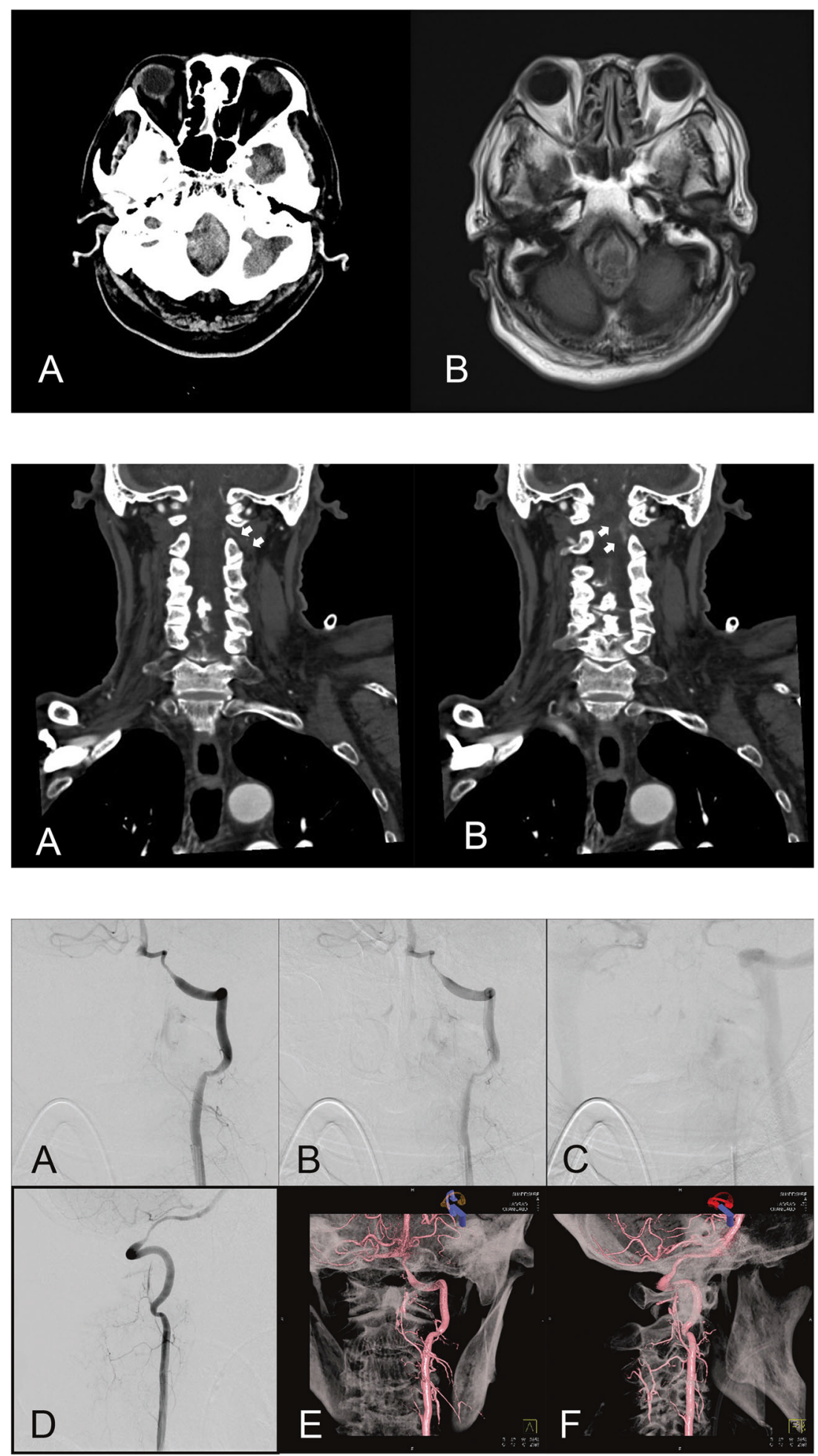

Neuroimaging (CT, MRI) in the emergency room revealed SAH at the CVJ (Fig. 1). ER doctors and neurosurgeons believed that the patient had suffered an intrinsic SAH with subsequent cardiopulmonary arrest. We performed CT angiography to identify the bleeding point, which revealed no aneurysms. Nevertheless, CT angiography showed a vessel running through the left $\mathrm{C} 2 / 3$ intervertebral foramen into the spinal canal (Fig. 2a). The ventral space of spinal cord revealed contrast enhancement ventrally. We believed there may have been an arteriovenous shunt, such as a dural arteriovenous fistula (Fig. 2b). We decided to perform angiography. 
Fig. 4 Plain CT at CVJ.

(a axial section, b sagittal section). The distance between the atlas and odontoid process in $7 \mathrm{~mm}$.

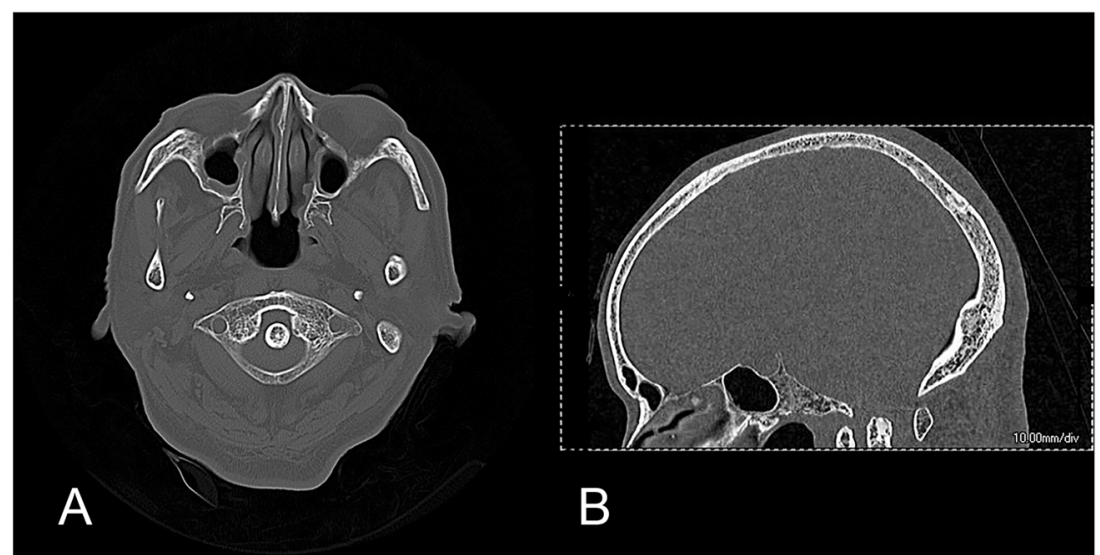

Fig. 5 CT angiography at CVJ. (a axial section, b sagittal section). The distance between the atlas and odontoid process in $1 \mathrm{~mm}$. Results differed from the plain $\mathrm{CT}$, and suggested atlantoaxial dislocation (AAD). Sagittal section revealed fused cervical subaxial vertebral bodies (C3-C7) with hyperostosis of the anterior longitudinal ligament.
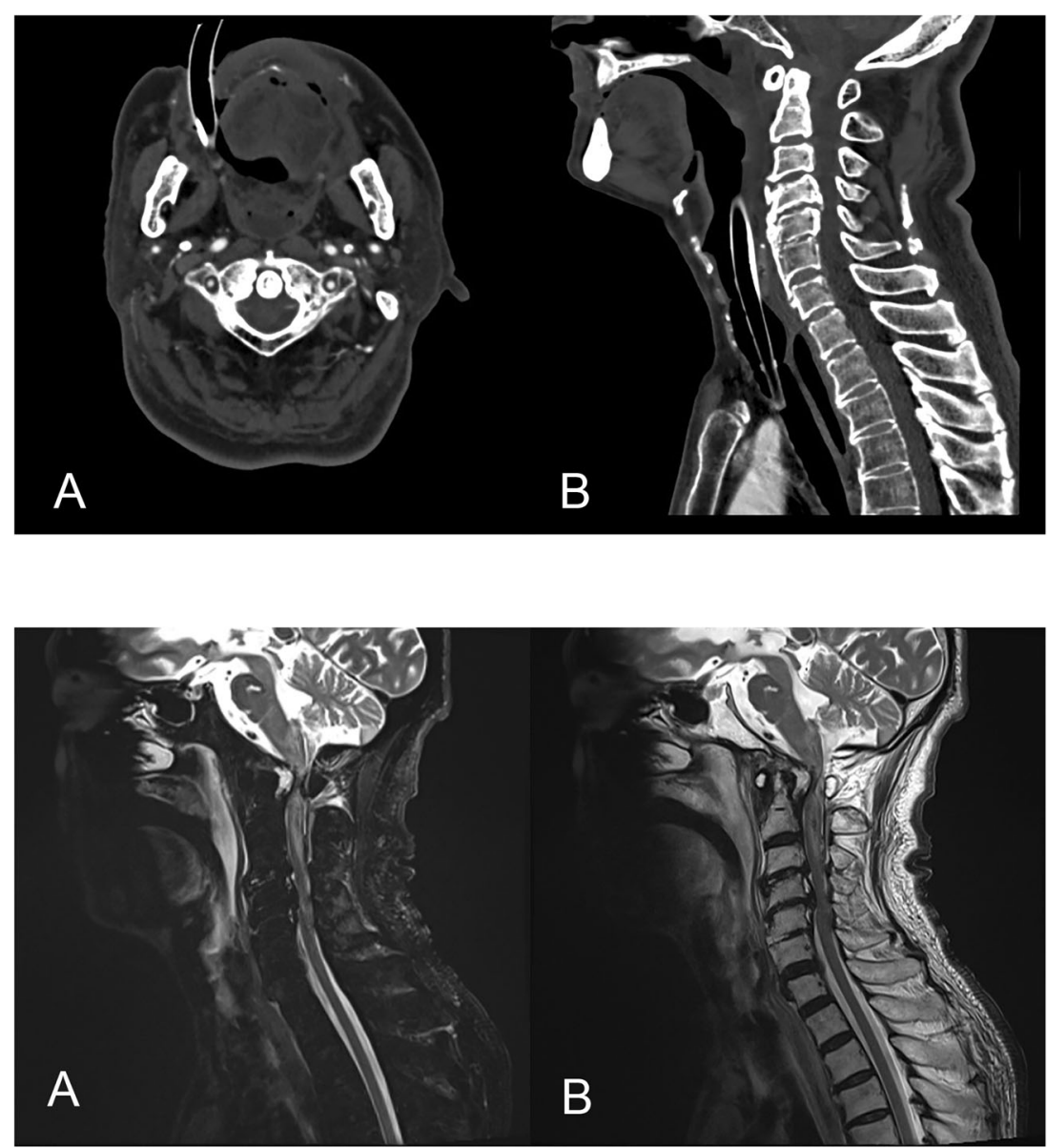

Fig. 6 Magnetic resonance imaging suggested trauma at CVJ. Cervical magnetic resonance imaging (a sagittal section of STIR, b T2 weighted image) revealing injury to the medulla oblongata and upper cervical spinal cord, with atlantoaxial dislocation and presence of a retrodental mass. The nuchal ligament was damaged at the $\mathrm{C} 1 / \mathrm{C} 2$ segment. There was also retropharyngeal hemorrhage.
Angiography of the left vertebral artery revealed extravasation from the spinal branch of the left vertebral artery, without venous filling in all phases (Fig. 3a-c). Angiographically occult vascular malformation could not be completely ruled out. However, this did not appear to be a vascular malformation with an arteriovenous shunt, but rather a traumatic laceration of the artery (Fig. 3).

The patient's consciousness gradually improved $12 \mathrm{~h}$ after admission. He was able to move his eyes on verbal command. However, spontaneous respiration never recovered and he was unable to move his extremities. We suspected that he suffered a spinal cord disorder or injury.

We compared axial and sagittal images of plain CT (Fig. 4) and CT angiography (Fig. 5). The distance between the atlas and odontoid process suggested AAD. A sagittal section of bone image of $\mathrm{CT}$ angiography revealed fused cervical subaxial vertebral bodies (C3-C7) with hyperostosis of the anterior longitudinal ligament (Fig. 5b). 
Cervical magnetic resonance images revealed injury to the medulla oblongata and upper cervical spinal cord, with $\mathrm{AAD}$ and presence of a retrodental mass (Fig. 6). The nuchal ligament was damaged at the $\mathrm{C} 1 / \mathrm{C} 2$ segment. Retropharyngeal hemorrhage was also observed. The patient had suffered from cerebral infarction 3 months prior. Scout image for cerebral MRI at that time did not reveal the retrodental mass (Fig. 7).

We believed he might have suffered CVJ trauma, traumatic SAH, and injury of the lower brain stem and upper cervical region. SCI might have originated from the AAD. The patient was in critical condition; nevertheless, his son requested that we administer all possible therapies. We planned a radiological interventional to stop the extra- and intra-dural bleeding. We also performed $\mathrm{C} 1$ laminectomy for decompression and removal of hematoma if identified. We did not perform a $\mathrm{C} 1 / 2$ fixation, though this would have been performed if his neurological state improved.

We performed embolization of the branch of left vertebral artery. We embolized and stopped the bleeding with

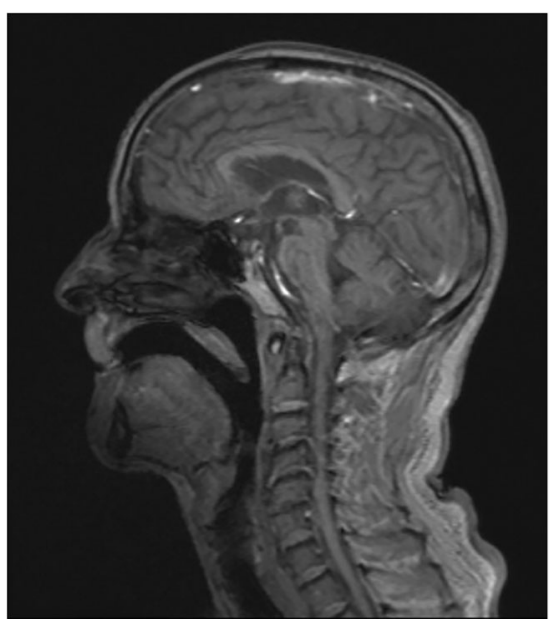

Fig. 7 Scout image for the cerebral MRI 3 months prior did not reveal the mass. coiling and n-butyl-2-cyanoacrylate (Fig. 8). Bilateral vertebral arteries were stenotic at the entry points of the dura (Fig. 8a). After embolization, we performed $\mathrm{C} 1$ laminectomy and an extra- and intra-dural inspection. Only SAH and contusion without epidural or subdural hematoma were found in the dorsal side of the spinal cord (Fig. 9a). Post operation, the patient was able to move his extremities slightly, and spontaneous respiration partially returned. Nevertheless, he remained bedridden on artificial ventilation, and died of pneumonia 87 days later.

\section{Discussion}

Traumatic atlantoaxial joint dislocation is a lethal injury. Survival after traumatic AAD is rare [1]. AAD without odontoid fracture is usually associated with rupture of the alar ligament [1]. Movement of the atlantoaxial joint is limited by the $\mathrm{C} 1-\mathrm{C} 2$ articulation, the ipsilateral transverse ligament, the contralateral alar ligament, and the capsular ligaments [2,3]. Our patient had a subligamentous hemorrhage. Therefore, this case should be defined as a traumatic AAD without fracture. In this case, subaxial cervical vertebral bodies were fused with ossification of the anterior ligament, and traumatic force might concentrate into the $\mathrm{C} 1-\mathrm{C} 2$ joint. One case exists of atlantoaxial subluxaton with diffuse idiopathic skeletal hyperostosis [4].

Traumatic AAD may cause respiratory arrest and arterial injuries. VA dissection may occur in the intra- and extra-cranial portion. Pull-out damage of the medullary branch in the hyperextension position occurs because the VA is fixed to $\mathrm{C} 1$ [5]. Other conditions may exist, including SAH, craniovertebral ligamentous disruption, retrodental epidural hematoma, and focal vertebral artery stenosis at the dural entry point.

Past case reports suggested that injury of the VA or its branches may cause SAH in CVJ with upper cervical trauma. Coast and Gee reported five autopsy cases after sudden death with blunt head trauma [6]. They found SAH in CVJ with VA lacerations just above the dural entry
Fig. 8 After embolization for the branch of the left vertebral artery. Bilateral vertebral arteries with stenosis at dural entry points.

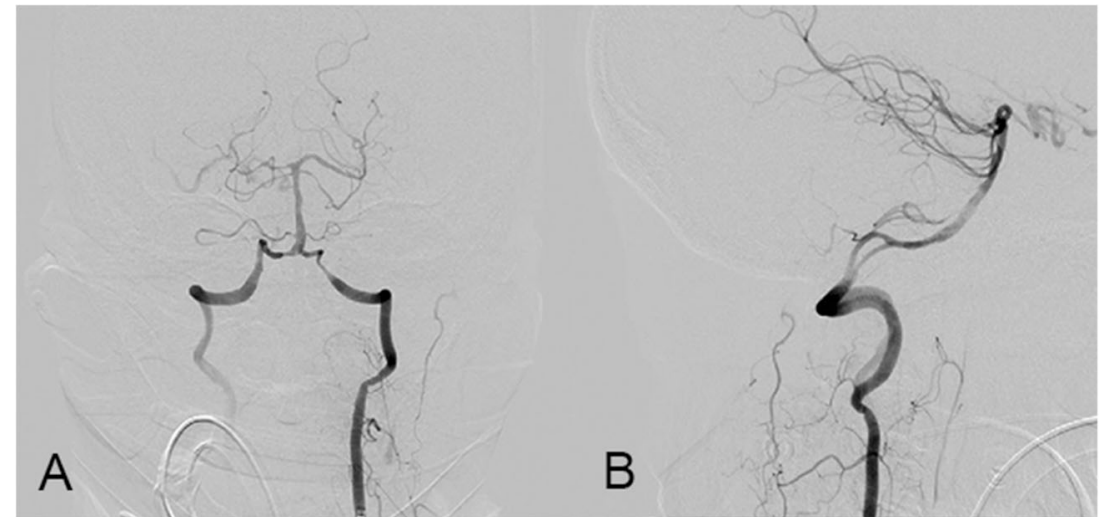


Fig. $9 \mathrm{C1}$ laminectomy and extra- and intra-dural inspection. a Photograph during surgery after $\mathrm{C} 1$ laminectomy and dural opening. Only SAH and contusion without epidural or subdural hematoma were found in dorsal side of spinal cord. b, c CT after surgery.

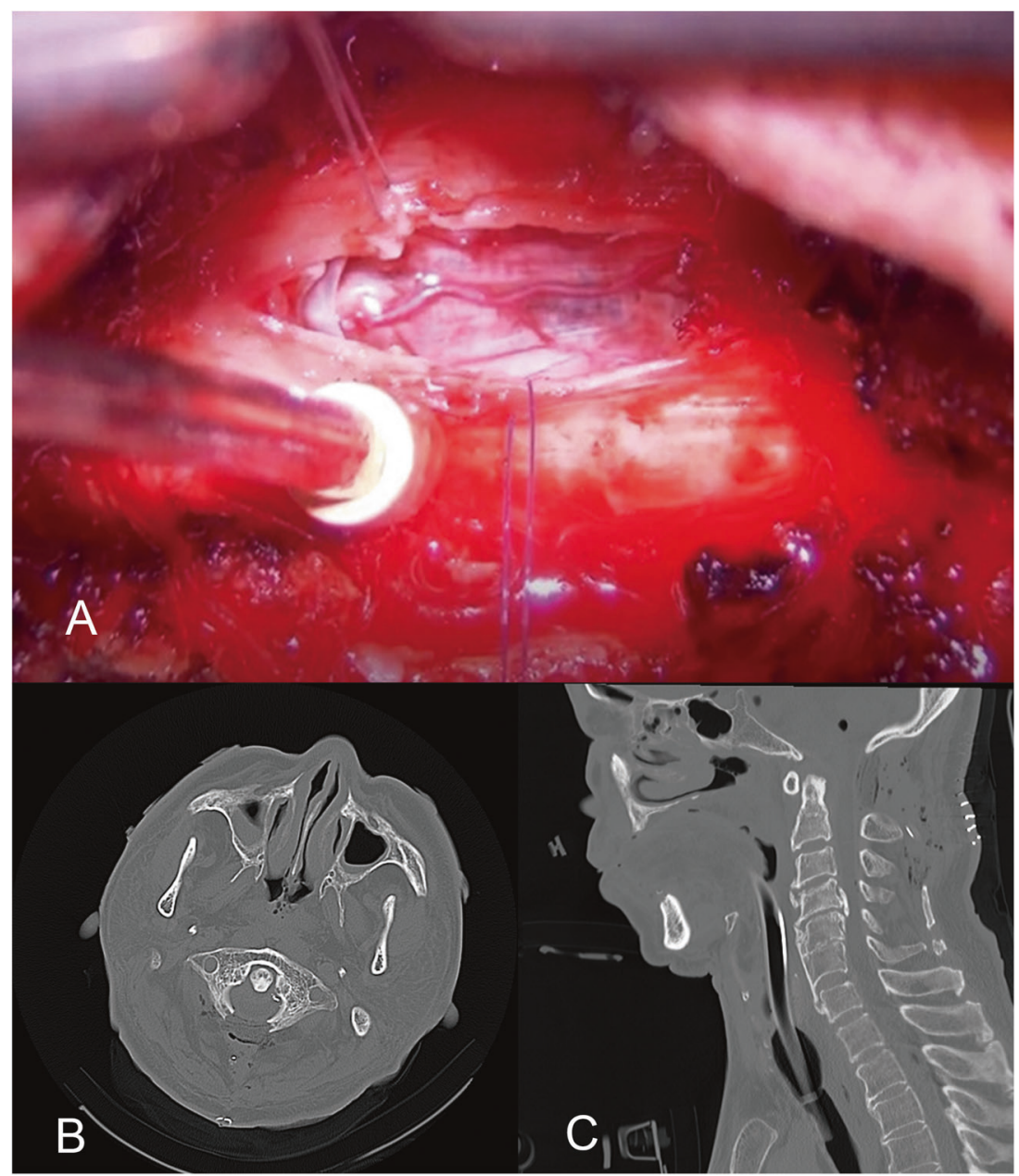

points. Harland reported 17 autopsy cases after SAH with upper cervical trauma [7]. Three pathologies of SAH have been hypothesized: (1) VA fixed by the transverse foramen of the atlas is subjected to shearing force, causing rupture of the vessel wall; (2) compression forces on the VA against the lower border of the posterior atlantooccipital membrane tears the artery or one or more of its branches. When the tearing point is extracranial, the blood reaches the subarachnoid space; this may occur when the dissection of vessel wall is through the foramen magnum, or there are tears of the small branches of the VA that anastomose with the anterior spinal artery; Our case is thought to belong to this type; (3) VA ruptures at an intracranial point, as Coast reported. Brain stem injury or dural tear may also be associated with SAH in CVJ [7]. In our case, laceration of a branch of the VA might have caused the SAH in the CVJ.

In the literature, we were unable to find a case of a resuscitated patient with traumatic AAD with SAH in the CVJ. Xu reported one case of traumatic AAD with SAH in CVJ [8]. In that case, the patient was injured in a traffic accident. He remained awake and alert with quadriplegia, suggesting SCI. CT scans at the emergency room showed obvious posterior dislocation at the atlantoaxial joint [8]. Gregory reported that atlantooccipital dislocation (AOD) is often associated with SAH in the CVJ (85\%), and only a few patients are diagnosed with AOD in the emergency department (14\%) [9]. In patients with SAH in the CVJ, $\mathrm{AAD}$, and AOD should be included in the differential diagnosis.

In conclusion, this is the first case report describing a patient that had been resuscitated from cardiopulmonary arrest induced by traumatic AAD with SAH. AAD should be included in the differential diagnosis in cases of SAH at the CVJ.

Acknowledgements We would like to thank Editage (www.editage.com) for English language editing.

\section{Compliance with ethical standards}

Conflict of interest The authors declare that they have no conflict of interest.

Publisher's note Springer Nature remains neutral with regard to jurisdictional claims in published maps and institutional affiliations. 


\section{References}

1. Meyer C, Eysel P, Stein G. Traumatic atlantoaxial and fracturerelated dislocation. Biomed Res Int. 2019;2019:5297950.

2. Steinmetz MP, Mroz TE, Benzel EC. Craniovertebral junction: biomechanical considerations. Neurosurgery. 2010;66:7-12.

3. Martin MD, Bruner HJ, Maiman DJ. Anatomic and biomechanical considerations of the craniovertebral junction. Neurosurgery. 2010;66:2-6.

4. Oostveen JC, van de Laar MA, Tuynman FH. Anterior atlantoaxial subluxation in a patient with diffuse idiopathic skeletal hyperostosis. J Rheumatol. 1996;23:1441-4.
5. Kleweno CP, Zampini JM, White AP, Kasper EM, McGuire KJ. Survival after concurrent traumatic dislocation of the atlantooccipital and atlanto-axial joints: a case report and review of the literature. Spine. 2008;33:659-62.

6. Coast GC, Gee DJ. Traumatic subarachnoid haemorrhage: an alternative source. J Clin Pathol. 1984;37:1245-8.

7. Harland WA, Pitts JF, Watson AA. Subarachnoid haemorrhage due to upper cervical trauma. J Clin Pathol. 1983;36:1335-41.

8. Xu Y, Li F, Guan H, Xiong W. Traumatic posterior atlantoaxial dislocation without associated fracture but with neurological deficit: a case report and literature review. Medicine. 2015;94:1-6.

9. Przybylski GJ, Welch WC. Longitudinal atlantoaxial dislocation with type III odontoid fracture. J Neurosurg. 1996;84:666-70. 